\title{
Optical spatial solitons and modulation instabilities in transparent entangled polymer solutions
}

\author{
Manos Anyfantakis, ${ }^{1,2}$ Benoit Loppinet, ${ }^{1, *}$ George Fytas, ${ }^{1,2}$ and Stergios Pispas ${ }^{3}$ \\ ${ }^{1}$ Foundation for Research and Technology of Hellas, Institute of Electronic Structure and Laser, Box 1527, \\ 71110 Heraklion Crete, Greece \\ ${ }^{2}$ Departments of Chemistry and Material Science, University of Crete, 71003, Heraklion, Crete, Greece \\ ${ }^{3}$ National Hellenic Research Foundation Vass., Theoretical and Physical Chemistry Institute, \\ 48 Vass. Constantinou Avenue, 11635 Athens, Greece \\ *Corresponding author: benoit@iesl.forth.gr
}

Received July 28, 2008; revised September 17, 2008; accepted October 11, 2008; posted October 17, 2008 (Doc. ID 99430); published November 25, 2008

Fully transparent nondilute polydiene solutions exhibit optical nonlinearities when irradiated by a lowpower $\mathrm{cw}$ laser in the visible. The formation of optical spatial solitons is imaged through phase contrast microscopy. Both $(2+1) D$ and $(1+1) D$ modulational instabilities are evidenced as $2 \mathrm{D}$ and $1 \mathrm{D}$ arrays of linear filaments formed by beam defocusing or by using a cylindrical lens. The origin of the nonlinearity remains elusive. (C) 2008 Optical Society of America

OCIS codes: $190.6135,160.5470$.

Optical spatial solitons (OSS) designate self-trapped optical beams that propagate with no diffraction [1]. They occur in a number of materials where optical nonlinearities of various origins create self-focusing that counterbalances the natural diffraction. OSS and related phenomena, such as optical filamentation, are subjects of intense research activities [1,2]. In condensed matter, OSS have been predicted and observed in nematic liquid crystals [3], photorefractive solids [4], and colloidal suspensions [5,6], where the light-induced change of the refractive index arises from variations of the molecular orientation, charge carrier, and colloid concentrations, respectively. Despite their very different microscopic origins and mechanisms, the nonlinearities lead to a similar phenomenology. In particular, modulational instabilities (MI) are observed that lead to the breakup of single soliton (filament) propagation into self-ordered OSS (multifilament) arrays in those media [7-10]. The established case of dielectric colloidal hard spheres dispersed in a dielectric fluid has recently received renewed attention and MI were demonstrated both theoretically [11] and experimentally [12]. Thermal effects may also be the source of optical nonlinearities that can be important in binary fluid mixtures, particularly near the phase separation [13-15], but to the best of our knowledge, thermally induced OSS have not been reported.

Recently, an anomalous case of optical pattern formation in polydiene solutions was reported $[16,17]$. Mild laser light irradiation of fully transparent entangled solutions of specific polydienes [1,4 polybutadiene (PB) and polyisoprene (PI)] were found to lead to a light induced matter organization. "Written" patterns along the propagation direction of a low-power (at milliwatt levels) cw laser were observed, arising from a local change of the refractive index [16]. This effect was also utilized to write holographic gratings [17]. Despite a detailed phenomenology the precise nature of the microscopic coupling between the light field and the local polymer structure still remains elusive.

In this Letter, we revisit this optical pattern formation and unambiguously establish through microscopy observations the OSS nature of the effect. Single filament formation was clearly imaged by phase contrast microscopy, which allowed quantification of the formation kinetics. The filaments could be as long as several centimeters. Furthermore, the occurrence of multifilament patterns arranged in both one and two dimensions, reminiscent of MI, was clearly demonstrated.

Filament formation and its simultaneous imaging were achieved using a simple setup (Fig. 1A). A homemade flat glass cell (C) 2 -mm-long ( $\sim 7 \times$ diffraction length) was mounted on an $(x, y)$-translation stage placed on a Zeiss Axioscop microscope for phase contrast imaging in the perpendicular $(z)$ direction. A cw laser with wavelength of $\lambda=671 \mathrm{~nm}$ and maximum power of $P=300 \mathrm{~mW}$ propagating along the $x$ direction was used as the "writing" radiation source. The laser beam was focused by a $4 \times$ microscope lens (Melles Griot) (L1) in the middle of the cell resulting
A

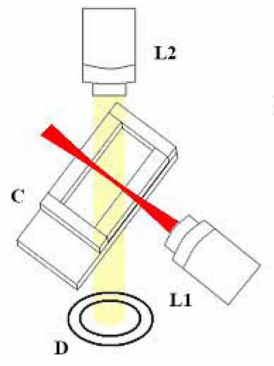

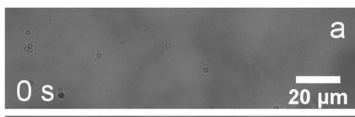
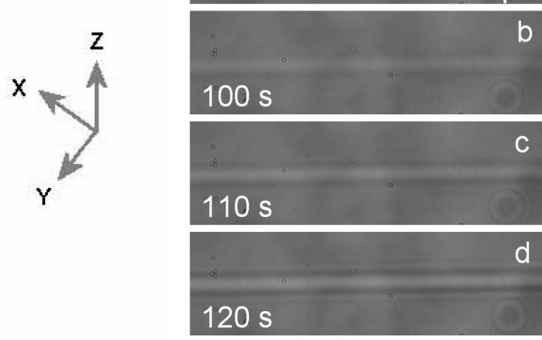

Fig. 1. (Color online) A, Schematic of the experimental setup. B (a-d), Phase contrast images of the filaments in a $2.42 \%$ PI $(N=15700)$-decane solution at different irradiation times $(\lambda=671 \mathrm{~nm}, P=29 \mathrm{~mW})$. 
in a focal spot with a diameter of $13 \mu \mathrm{m}$. Real-time imaging was implemented by means of the whitelight Köhler illumination unit of the microscope to create a collimated beam of a few millimeters $(D)$, a $20 \times$ objective lens (Zeiss) (L2), and a $640 \times 480$ CCD camera (Unibrain) fitted with the movable appropriate filter. The pictures were acquired with the microscope objective lens slightly defocused $(\sim 40 \mu \mathrm{m})$ to permit quantitative phase contrast analysis where the intensity measured at the CCD relates to the refractive index profile [18]. The samples consisted of well-equilibrated concentrated solutions (in the range $2-15 \mathrm{wt} . \%$ ) of commercial cis-1,4 PB $\left(-\mathrm{CH}_{2}-\mathrm{CH}=\mathrm{CH}-\mathrm{CH}_{2}-\right)_{N}$ and anionically polymerized monodispersed cis-1,4 PI $\left(-\mathrm{CH}_{2}-\mathrm{C}\left(\mathrm{CH}_{3}\right)=\mathrm{CH}-\mathrm{CH}_{2}-\right)_{N}$ in different alkanes (tetradecane, decane) where the degree of polymerization $N$ varies between 1000 and 16,000 .

Figure 1B (a-d) presents typical phase contrast images acquired at different times of laser light irradiation of a $2.42 \% \mathrm{PI}(N=15,700)$-decane solution using a power of $29 \mathrm{~mW}$. The development of a fiberlike pattern in the propagation direction of the laser beam was clearly imaged over the whole field of view. Notably, the lateral dimension of the formed filament remained virtually constant, whereas the contrast increased with the irradiation time. A better quantification of the observations was obtained through the intensity profile across the fiber $I(y, t)$ averaged over a portion $(\sim 30 \mu \mathrm{m})$ of the fiber length. The intensity profiles [normalized by $I(t=0)$ ] at different elapsed irradiation times $t$ are presented in Fig. 2A. Assuming Gaussian refractive index profiles, the full width at $I(y, t)=0$ is proportional to the diameter $D(t)$. An almost constant diameter $D \sim 8 \mu \mathrm{m}$, clearly smaller than the beam waist $(13 \mu \mathrm{m})$, was obtained. Filaments formed at different powers assumed similar $D$ values. The self-guiding image through scattered intensity confirmed the reduced fiber size compared to the beam waist.

The intensity maximum in the center of the fiber $I_{\max }=I(y=0)$ is proportional to the refractive index gradient over the filament cross section $2 \Delta n(t) / D(t)$, where $\Delta n$ represents the refractive index increment in the center of the filament compared to nonirradiated regions [18].
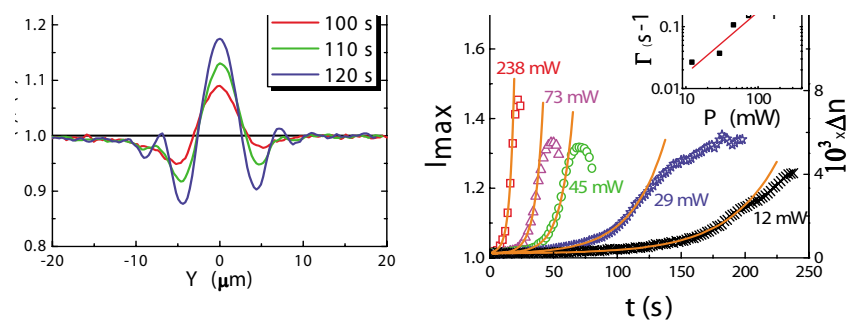

Fig. 2. (Color online) A, Image intensity profiles $I(t) / I(0)$ across the fibers for different writing (laser-medium interacting) times relative to $I(0)$ at $t=0$. B, The maximum $I_{\max }=I(t) / I(0)$ versus the function of the irradiation time for laser power $P$. Solid curves are exponential growth fits to the data. Right $y$ axis: refractive index contrast $\Delta n$. Inset: growth rate versus the $P$.
The growth $I_{\max }$ with irradiation time for a $2.42 \%$ PI $(N=15,700)$-decane solution is shown in Fig. 2B for different $P$ s. Obviously, the filament formation speeds up with increasing $P$. The early increase of $I_{\max }(t)$ is well represented by the exponential growth, $I_{\max }(t)=A \exp (\Gamma t)$. At longer irradiation times, a deviation from the exponential growth was observed reaching eventually saturation or even decrease of the contrast. The observed decays (Fig. 2B) were attributed to small variations of the filament position in a solution with relatively low viscosity. The growth rate $\Gamma$ was found to exhibit a nearly linear increase with $P$ (inset of Fig. 2B). Estimates of the refractive index contrast $\Delta n$ between the center of the fiber and the nonirradiated region were deduced [18] from the value of $I_{\max }$, through the relation $\Delta n=\left(I_{\max }-1\right) / 2^{*} \pi^{1 / 2}(\Delta z / D)$ obtained for a Gaussian-like profile where $\Delta z$ is the defocus distance. The computed $\Delta n$ using $D=8 \mu \mathrm{m}$ and $\Delta z$ $=40 \mu \mathrm{m}$ plotted in Fig. $2 \mathrm{~B}$ (right $y$ axis) fell in the $10^{-3}$ range. If $\Delta n$ originated from an increase of polymer concentration $\Delta c$, then $\Delta c$ is easily estimated since $\Delta n=(\mathrm{d} n / \mathrm{d} c) \Delta c$. Using for the refractive index increment $(\mathrm{d} n / \mathrm{d} c) \sim 0.1 \mathrm{ml} / \mathrm{g}$, a typical value for PI $(n=1.52)$-decane $(n=1.41)$ solution, $\Delta c$ went up to $10 \%$. This significant increase of the local concentration in an overall $2.4 \%$ PI-decane solution still remains far from the maximum of pure polymer.

This single filament formation as described above is very reminiscent of OSS. The correspondence to OSS is strengthened by another striking observation: the formation of a bundle array of regularly spaced parallel filaments oriented along the propagation direction when the writing lens is defocused (Fig. 3A). A further demonstration of multifilament formation is obtained when the lens L1 is replaced by a cylindrical lens (focal distance $\sim 1 \mathrm{~cm}$ ), producing a sheet of light into the imaged solution. It gives rise to the formation of up to 20 observable filaments regularly spaced in one plane as seen in Figs. 3B and 3C. The waveguiding by an individual filament was clearly seen on the scattering image Fig. 3B. The different filaments appear to have the same transverse dimension, very similar to that of the single filament formed with the lens L1 in focus. Multifilament patterns are usually explained as a result of MI, i.e., small wavefront perturbations that cause the optical field to break up into a periodic array [8-10]. The present polydiene solutions therefore provide a very

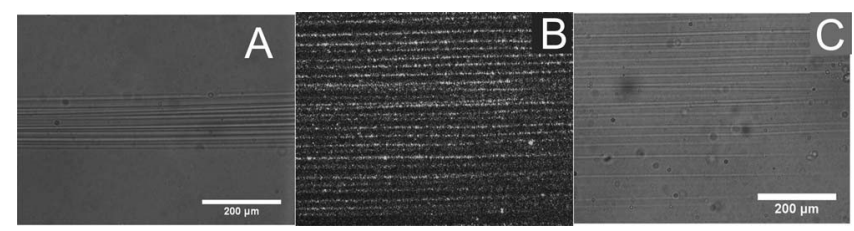

Fig. 3. Filament arrays in a $\mathrm{PB}(N=7100)$-tetradecane $(n=1.43)$ solution, $c=14.7 \%$. A, $(2+1) D$ OSS phase contrast image of the filaments bundle crated with defocused L1 $(P=300 \mathrm{~mW} 600 \mathrm{~s})$. B and C $(1+1) D$ OSS planar array of filaments created with a cylindrical lens $(P=300 \mathrm{~mW}$, $900 \mathrm{~s})$. B, Laser light self-guiding observed through scattered light. $\mathrm{C}$, phase contrast image. 
clear example, the first to the best of our knowledge, of a binary mixture where MI can be clearly observed with both $(2+1) D$ and $(1+1) D$ solitons.

Inevitably, one has to address the origin of such a clear nonlinear optical phenomenology at low power levels, i.e., the nature and the origin of the optical nonlinearity. Despite the very clear observation the phenomenon resists standard explanation. The increase of the refractive index undoubtedly relates to an increase of the polymer concentration in regions of large laser intensity. It is therefore tempting to attribute it to electrostriction. However, owing to the low $\mathrm{d} n / \mathrm{d} c$ and the small characteristic length of semidilute solutions (a few nanometers) the electrostrictive effect at work in the hard sphere colloidal dispersion is not expected to lead to measurable refractive index changes. Indeed an optical trapping phenomenon in polymer solutions has never been reported. Likewise, the very weak absorption is not expected to initiate either any large concentration variations [13], as solutions are in good solvents far from any demixing, or photopolymerization effects [19]. Obviously some more complex mechanisms are at work, which require the specific chemical structure of the polymer backbone. Stimulated Raman scattering, cis-trans conformation changes are among the exotic candidates that could lead to such increase of polymer concentration in the focus of the laser beam and are currently under investigation.

The above observations clearly point toward a nonlocal and noninstantaneous light-matter interaction, where entangled polymer solutions specific properties, e.g., nonlinear mechanical response, might play a role. As to the stability of the formed filaments after switching off the irradiating laser beam, it was found to depend on the irradiation time. When the beam is stopped at early times, the filament will eventually fade away or break up into a linear array of dots. However, prolonged irradiation times lead to longlived filaments, and their fading out may easily last very long, i.e., months.

To summarize, OSS and MI have been clearly demonstrated. The polydiene solutions offer an unexpected, simple, and versatile system for nonlinear optics studies, with, however, unclear physical origins.

The Greek General Secretariat for Research and Technology (program PENED-03ED805 and the 6th
Research Framework Program of the European Union (EU) (SoftComp Network of Excellence) are acknowledged for financial support. It is a pleasure to thank S. Coppola for the kind supply of samples and C. Mantzaridis for assistance in synthesis; R. Sigel, N. Vainos, and D. Papazoglou for many useful discussions; and, finally, many of the laboratory visitors for their genuine interest in this surprising effect.

\section{References}

1. G. I. Stegeman and M. Segev, Science 286, 1518 (1999).

2. A. Couairon and A. Mysyrowicz, Phys. Rep. 441, 47 (2007).

3. M. Peccianti, C. Conti, C. Assanto, A. De Luca, and C. Umeton, Nature 432, 733 (2004).

4. M. Shih, P. Leach, M. Segev, M. H. Garrett, G. J. Salamo, and G. C. Valley, Opt. Lett. 21, 324 (1996).

5. A. Askin, J. M. Dziedzic, and P. W. Smith, Opt. Lett. 7, 276 (1982).

6. V. E. Yashin, S. A. Chizhov, R. L. Sabirov, T. V. Starchikova, N. V. Vysotina, N. N. Rozanov, V. E. Semenov, V. A. Smirnov, and S. V. Fedorov, Opt. Spectrosc. 98, 466 (2005).

7. D. Kip, M. Soljacic, M. Segev, E. Eugenieva, and D. N. Christodoulides, Science 290, 495 (2000).

8. R. El-Ganainy, D. N. Christodoulides, Z. H. Musslimani, C. Rotschild, and M. Segev, Opt. Lett. 32, 3185 (2007).

9. R. El-Ganainy, D. N. Christodoulides, C. Rotschild, and M. Segev, Opt. Express 15, 10207 (2007).

10. M. Matuszewski, W. Krolikowski, and Y. S. Kivshar, Opt. Express 16, 1371 (2008).

11. C. Conti, N. Ghofranha, G. Ruocco, and S. Trillo, Phys. Rev. Lett. 97, 123903 (2006).

12. P. J. Reece, E. M. Wright, and K. Dholakia, Phys. Rev. Lett. 98, 203902 (2007).

13. S. Juodkazis, N. Mukai, R. Wakaki, A. Yamaguchi, S. Matsuo, and H. Misawa, Nature 408, 178 (2000).

14. S. Buil, E. Hugonnot, and J. P. Delville, Phys. Rev. E 63, 041504 (2001).

15. A. Voit, A. Krekhov, and W. Koehler, Phys. Rev. E 76, 011808 (2007).

16. R. Sigel, G. Fytas, N. Vainos, S. Pispas, and N. Hadjichristidis, Science 297, 67 (2002).

17. B. Loppinet, E. Somma, N. Vainos, and G. Fytas, J. Am. Chem. Soc. 127, 9678 (2005).

18. A. Barty, K. A. Nugent, D. Paganin, and A. Roberts, Opt. Lett. 23, 817 (1998).

19. A. S. Kewitsch and A. Yariv, Opt. Lett. 21, 24 (1996). 